\title{
VARIACIONES ESPACIALES EN LAS COMUNIDADES DE MACROINVERTEBRADOS DEL RÍO AGÜERA (PAÍS VASCO- CANTABRIA) EN DOS ÉPOCAS CON DIFERENTES CONDICIONES DE RÉGIMEN HIDROLÓGICO
}

\author{
P. Riaño, A. Basaguren, J. Pozo \\ Lab. Ecología, F. Ciencias, Universidad del País Vasco/ E.H.U., \\ Apdo. 644,48080 Bilbao, Spain
}

Palabras clave: macroinvertebrates, discharge, stream ecosystem.

\author{
ABSTRACT \\ SPATIAL CHANGES OF MACROINVERTEBRATE COMMUNITIES IN THE AGÜERA STREAM (BASQUE \\ COUNTRY-CANTABRIA, NORTHERN SPAIN) UNDER TWO DIFFERENT SEASONAL HYDROLOGIC REGIMES
}

Macroinvertebrate communities along the Agüera stream (Basque Country-Cantabria, Northem Spain) were studied in autumn 1988 and spring 1989. A clear downstream increase in density occurs under low flows, but this pattem is disrupted at moderately high discharges. Collector-gatherers and generalists (mainly chironomids) alternate their dominance spatially, generalists being more important in eutrophicated reaches. A spatial segregation is also shown by shredders (more important under low disturbed forested reaches), scrapers (increasing with periphyton) and collector-filterers (related to the eutrophication of waters caused by human settlements). Hydrologic regime, periphyton biomass and disturbance by urban sewage seem to be the main factors responsible for the spatial distribution and abundance of macroinvertebrate communities in the Agüera stream.

\section{INTRODUCCIÓN}

Durante los últimos años se han incrementado los trabajos dedicados a observar la influencia que tienen las variables tanto bióticas como abióticas sobre la organización de las comunidades de macroinvertebrados de los sistemas fluviales (HAWKINS \& SEDELL, 1981; HART, 1983; OUTRIDGE, 1988; RESH et al., 1988; ROSILLON, 1989; TOWNSEND, 1989; PECKARSKY et al., 1990; RICHARDSON, 1991; WARD, 1992).

Una de las teorías, compartida o no, que ha suscitado gran número de trabajos ha sido la del River Continuum (RCC) de VANNOTE et al. (1980), según la cual, en los sistemas fluviales se establece un gradiente contínuo de condiciones y de recursos desde los tramos de cabecera hasta la desembocadura, que determinan la estructura de las comunidades de macroinvertebrados del bentos fluvial. Para dar significado a algunos de los presupuestos de esta teona, los macroinvertebrados fluviales se consi- deran clasificados en grupos funcionales según su modo de alimentación (CUMMINS, 1974; MERRITT \& CUMMINS, 1978; CUMMINS \& KLUG, 1979). Estos organismos pueden asignarse a seis categorías tróficas: fragmentadores, que procesan principalmente la materia orgánica particulada gruesa (MOPG) en forma de hojarasca; filtradores, que filtran la materia orgánica particulada fina (MOPF) suspendida en el agua; recolectores que recogen la MOPF del sedimento; raspadores del substrato, con adaptaciones morfológicas y etológicas para raspar el alimento adherido a superficies, particularmente perifiton; depredadores, adaptados para la captura de presas vivas, y generalistas.

En este trabajo, se describen los cambios espaciales y temporales (en la composición taxonómica y estructura trófica) que sufre la comunidad de macroinvertebrados a lo largo del gradiente fluvial. Se inserta dentro del estudio global sobre la cuenca fluvial del río Agüera, de corto recomdo y poco alterado en comparación con otros ríos de Bizkaia (EUSKOIKER, 1988; ORIVE et al, 1989). 


\section{ÁREA DE ESTUDIO}

El área de estudio corresponde a la cuenca del no Agüera que se localiza al norte de la Península Ibérica, entre las provincias de Bizkaia y Cantabria (Fig. 1). Las coordenadas U.T.M. son 30T VN 7883, en el nacimiento y 30T VP 7403, en la desembocadura. El Agüera es un río de $30 \mathrm{~km}$. de recomdo al que vierten sus aguas pequeños afluentes que drenan una superficie de $145 \mathrm{~km}^{2}$, escasamente poblada y de dedicación basicamente agropecuaria, en la que apenas existe actividad industrial.

Geológicamente, es, en su mayor parte, de naturaleza silícea, exceptuando una amplia zona central y parte de la desembocadura de naturaleza caliza.

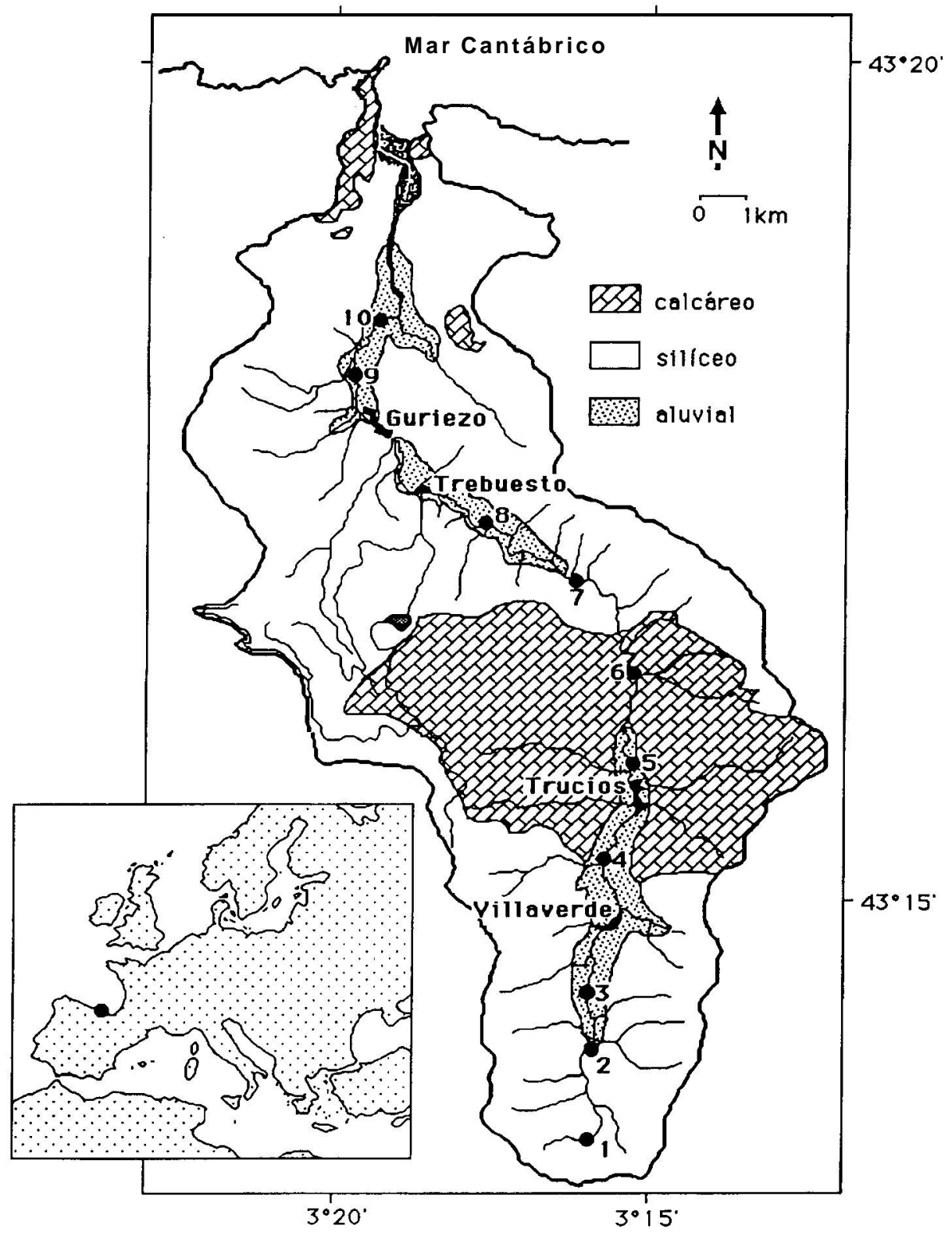

FIGURA 1. Cuenca del río Agüera. Características geológicas, núcleos urbanos y localización de las estaciones de muestreo FIGURE 1. The Agüera stream catchment. Main geological features, human settlements, and location of sampling sites. 
El clima puede considerarse templado húmedo, con lluvias abundantes durante todo el año. Sin embargo, en la época de muestreo (otoño de 1988 y primavera de 1989), el régimen térmico y de precipitaciones, se vio alterado respecto a lo habitual. Durante el otoño se registraron temperaturas moderadamente altas y escasez de lluvias $\left(12.8^{\circ} \mathrm{C}\right.$ y $22.8 \mathrm{~mm}$. de temperatura media y precipitación, respectivamente, en Noviembre de 1988). En primavera se produjeron fuertes lluvias que se reflejaron en unos mayores caudales $\left(10.6{ }^{\circ} \mathrm{C}\right.$ y $345.3 \mathrm{~mm}$. de temperatura media y precipitación,

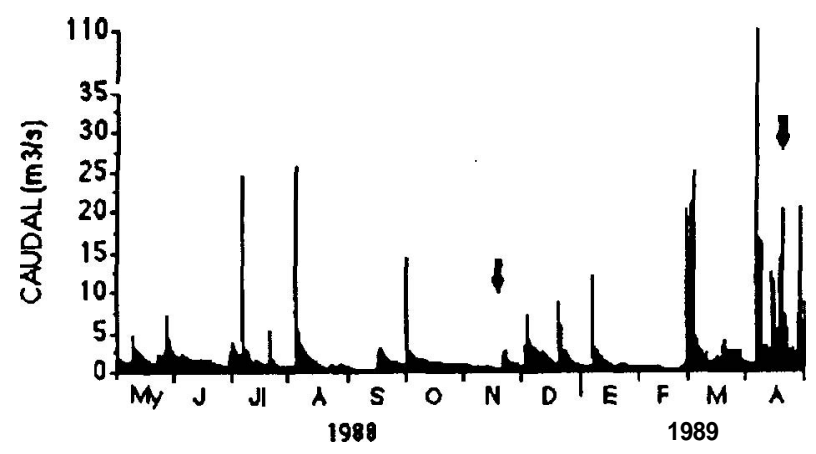

FIGURA 2. Descarga fluvial en el río Agüera (Mayo 88 - Abril 89) medida en la estación de aforo de Guriezo. Las flechas señalan las fechas de muestreo.

FIGURE 2. Daily discharge in the Agüera stream (May 88 - April 89) measured in Gunezo. Arrows indicate dates of sampling. respectivamente, en Abril de 1989). El hidrograma del ciclo anual dentro del cual está incluído el periodo de estudio se presenta en la Fig. 2 (datos tomados en la estación de Guriezo y cedidos por la Confederación Hidrográfica del Norte).

La vegetación dominante es de bosque mixto caducifolio en la cabecera, predominando en el resto de la cuenca las plantaciones de pinos y eucaliptos, junto a los prados y cultivos. En la zona central se da el encinar. Las orillas del río están dominadas por alisedas en estado variable de conservación.

Como ha sido descrito ya en un trabajo anterior por ELOSEGUI \& POZO (1992), el tramo alto del Agüera, poco alterado por la actividad humana, presenta baja mineralización y escasa concentración de nutrientes; el substrato calcáreo y los núcleos urbanos de tramos medios determinan el aumento de la mineralización y eutrofización; aguas abajo de Trucíos se produce un proceso de autodepuración al disminuir la intensidad de las actividades humanas, descendiendo así la concentración de nutrientes; en el tramo más bajo, los vertidos urbanos de Guriezo producen un nuevo aumento de la concentración de nutrientes y de materia orgánica particulada fina (MOPF). En la Tabla 1 se resumen algunas de las características físico-químicas de las estaciones en el periodo comprendido entre Noviembre del 88 y Abril del 89, en base a datos obtenidos con periodicidad quincenal.

TABLA 1. Rangos de algunas características físico-químicas del do Agüera entre Noviembrede 1988 y Abril de 1989. M=máximo, m=mínimo.

TABLE 1. Ranges of selected physical-chemical characteristics of the Agüera stream. Recorded period November 1988 - April 1989 . M=maximum, m=minimum.

VARIABLE RANGO

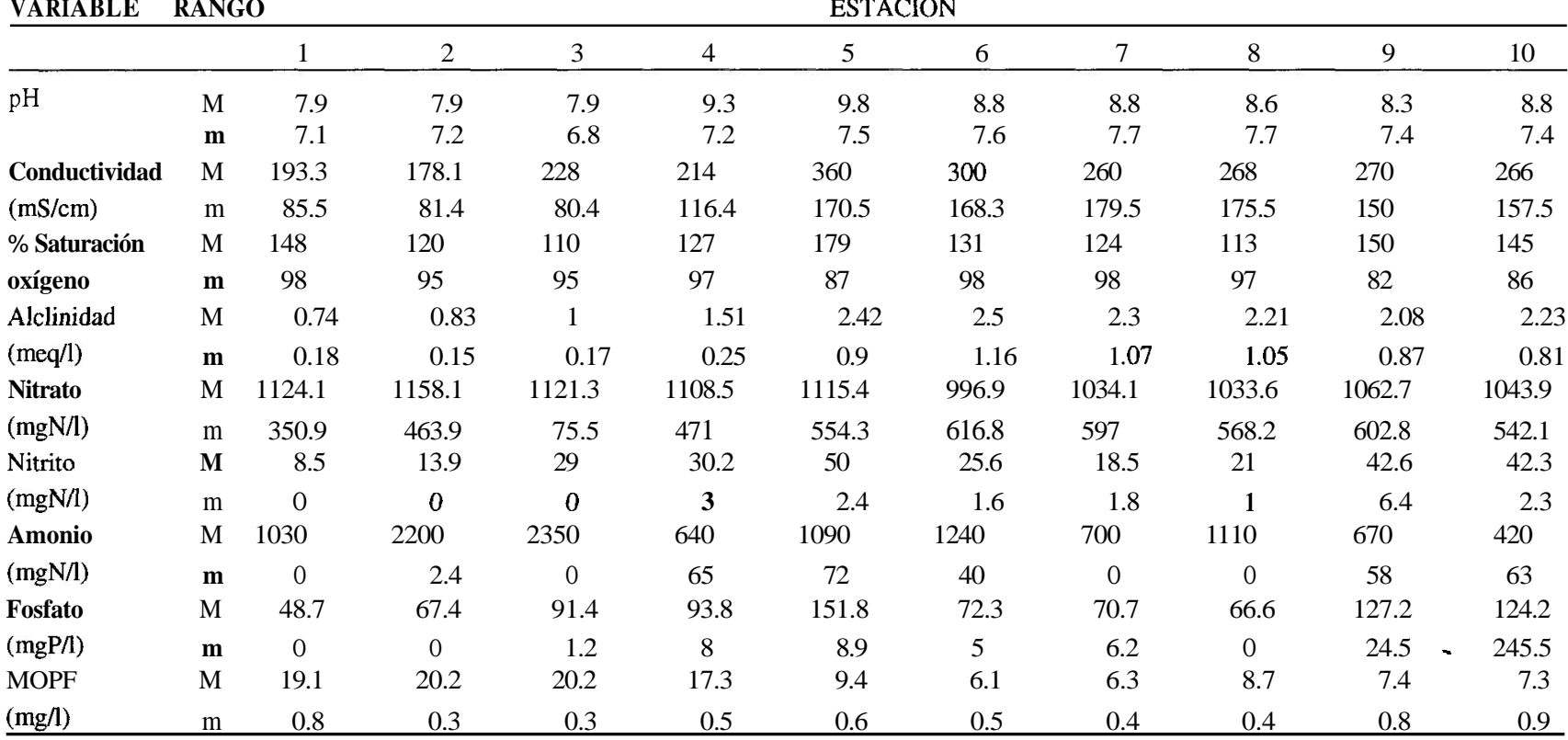




\section{MATERIAL Y MÉTODOS}

Se realizaron dos campañas de recogida de macroinvertebrados: una durante el otoño (Noviembre de 1988) y otra durante la primavera (Abril de 1989). Las muestras se tomaron en 10 estaciones situadas en el eje longitudinal del río Agüera, recogiéndose, en cada uno de los puntos, 5 réplicas mediante una red Surber de 200 pm. de tamaño de malla. Las muestras se lavaron "in situ" mediante un sistema de tamices de Icm. y 200 $\mu \mathrm{m}$. de poro respectivamente y fueron fijadas con formol al $5 \%$. En el laboratorio, la muestra se volvía a lavar en tamices de $500 \mu \mathrm{m}$. y $200 \mu \mathrm{pm}$. La submuestra de mayor tamaño era separada en su totalidad, mientras que de la inferior se tomaba una alícuota de 1/8. Los organismos fueron conservados en alcohol al $70 \%$ para su posterior identificación y contaje. No pudieron obtenerse los datos referentes a las estaciones 8 y 10 del muestreo de Abril

La significación estadística de las diferencias se ha llevado a cabo mediante análisis de la varianza y test de la $t$ (ZAR, 1984).

\section{RESULTADOS}

De forma general, en la figura 3 puede apreciarse que las comunidades de macroinvertebrados fueron más densas en otoño.

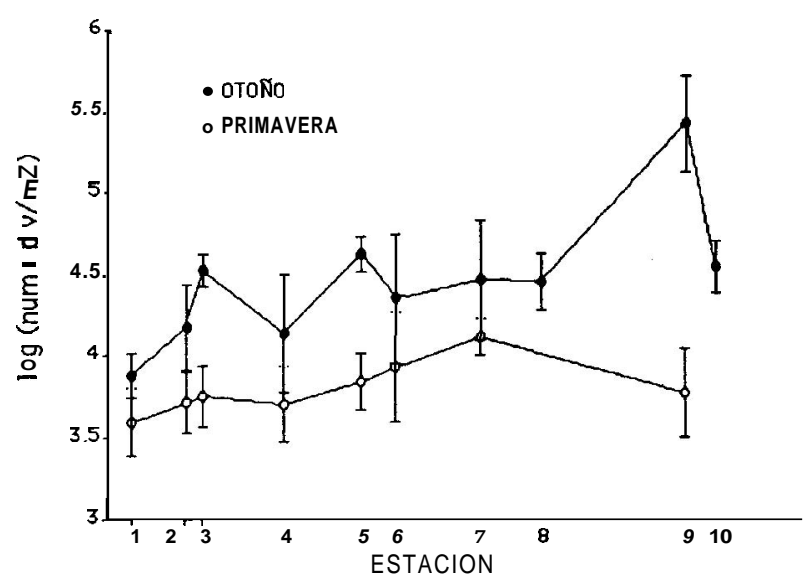

FIGURA 3. Variaciones longitudinales en la densidad de macroinvertebradosen otoño y primavera $(\overline{\mathrm{X}} \pm \mathrm{SD})$.

FIGURE 3. Longitudinal changes in density of macroinvertebrates in autumn and spring $(\overline{\mathrm{X}} \pm \mathrm{SD})$.

Durante esta época, en el eje longitudinal del Agüera se establece una clara diferenciación (ANOVA, p<0.01) entre los tramos superiores (estación 1) y los inferiores (estación 9), donde se da una mayor densidad (319.540 individuos $\left./ \mathrm{m}^{2}\right)$. En prima-

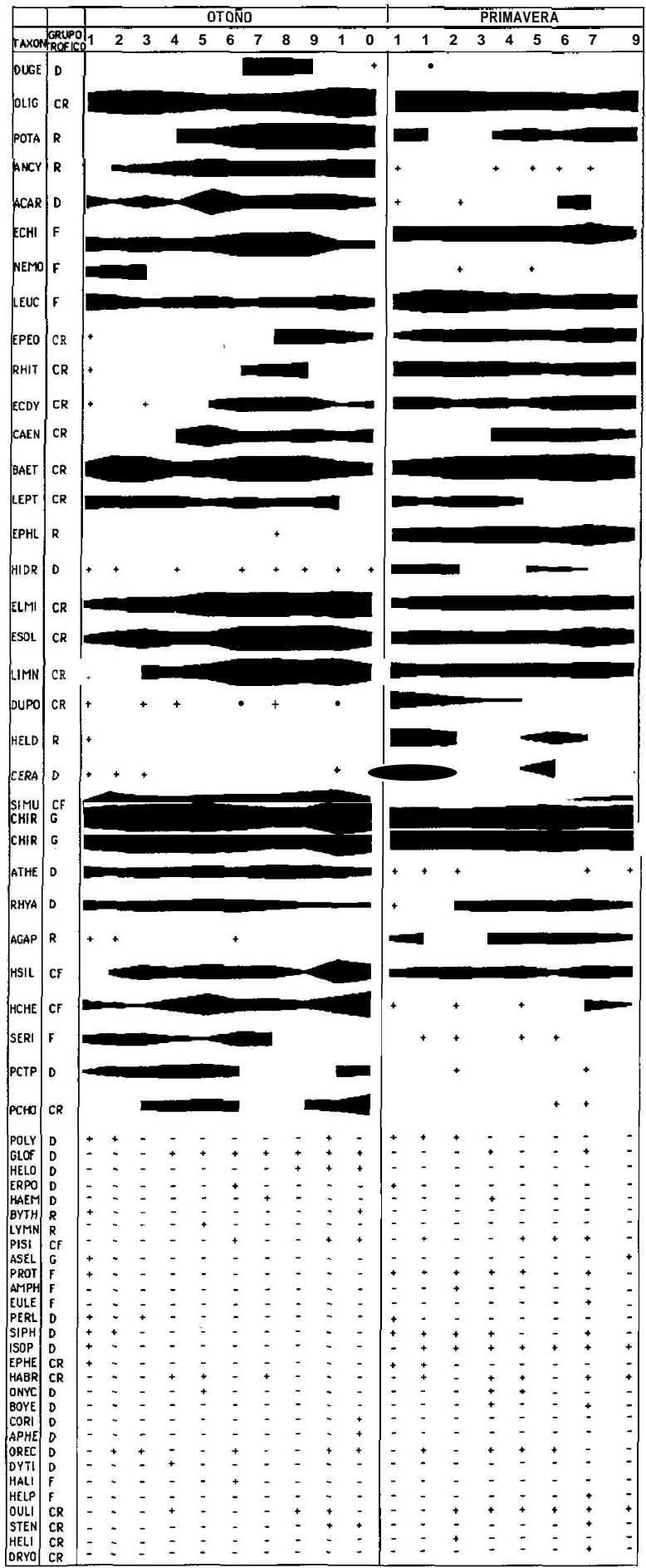

FIGURA 4. Distribución de densidades y/o presencia/ausencia de los taxones de macroinvertebradosen el río Agüera. Código en apéndice.

FIGURE 4. Distribution of densities and/or presence/absence of the macroinvertebrates taxa in the Agüera stream. See appendix for code. 


\begin{tabular}{|c|c|c|c|c|c|c|c|c|c|c|c|c|c|c|c|c|c|c|c|}
\hline & & & & & & OTO & & & & & & & & & PRII & IAVE & & & \\
\hline$T A \times O A$ & Gerup & & 2 & 3 & 4 & 5 & 6 & 7 & $\theta$ & 91 & & 1 & 2 & 3 & 4 & 5 & 6 & 7 & 9 \\
\hline \begin{tabular}{|} 
TPu \\
DXXI
\end{tabular} & $\underset{C R}{C R}$ & & $\overline{-}$ & E & $:$ & $=$ & $\overline{-}$ & - & $=$ & I & 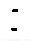 & $":$ & 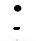 & $\dot{0}$ & $:$ & $=$ & & $\pi$ & $\overline{-}$ \\
\hline 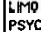 & $\begin{array}{l}D^{2} \\
c .\end{array}$ & & \pm & \pm & $:$ & : & $:$ & $\overline{-}$ & \pm & $=$ & $:$ & I: & $:$ & $:$ & & : & & $=$ & \\
\hline $\mid \begin{array}{l}\text { STRA } \\
\text { ETPPI }\end{array}$ & E & & : & $\overline{7}$ & $:$ & $\overline{+}$ & $:$ & $\overline{+}$ & $\overline{-}$ & $\overline{7}$ & $i$ & $i+$ & i & $=$ & $=$ & $\overline{-}$ & $=$ & $\bar{I}$ & $=$ \\
\hline $\begin{array}{l}\text { ATBA } \\
\text { ATRI }\end{array}$ & 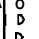 & & $\bar{z}$ & E & : & \pm & $:$ & : & $:$ & \pm & $\vdots$ & $\bar{z}$ & \pm & $\bar{z}$ & $\bar{z}$ & - & $\vdots$ & $\bar{z}$ & - \\
\hline BLEP & 票 & & $=$ & $=$ & - & $=$ & 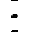 & $=$ & $=$ & $=$ & 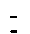 & : & $z$ & \pm & $=$ & $=$ & : & \pm & $=$ \\
\hline GLOS & R & & $=$ & & 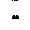 & $=$ & $=$ & - & - & ! & - & 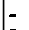 & 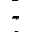 & $=$ & 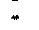 & $=$ & - & - & - \\
\hline Tita & $s$ & & $=$ & $=$ & 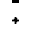 & 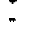 & + & + & $\bar{z}$ & + & + & $i$ & $=$ & $=$ & $=$ & 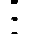 & $=$ & $\approx$ & 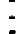 \\
\hline ODOA & F & \pm & - & : & $=$ & $=$ & $\overline{-}$ & $:$ & $=$ & $=$ & 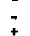 & 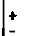 & $\overline{7}$ & $\mp$ & $=$ & $=$ & z & $\overline{7}$ & $=$ \\
\hline 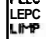 & CR & $\div$ & 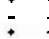 & $=$ & $\div$ & 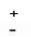 & $\bar{\vdots}$ & $\bar{z}$ & \pm & $=$ & : & 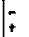 & 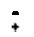 & $\mp$ & I & $\overline{-}$ & i & + & $=$ \\
\hline$\left.\right|_{\mathrm{PHO}} ^{\mathrm{CHIM}}$ & $c F$ & : & $\bar{t}$ & : & : & - & : & - & : & : & : & 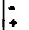 & \pm & : & : & : & : & : & : \\
\hline $\mid \begin{array}{ll}\text { EPI } \\
\text { GOER }\end{array}$ & F & \pm & : & $=$ & - & $\approx$ & + & \pm & \pm & $=$ & $=$ & $:$ & $=$ & $=$ & I & 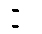 & - & 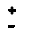 & $=$ \\
\hline $\begin{array}{l}\text { Nume } \\
\text { TAXO }\end{array}$ & & $4 z 2$ & $\begin{array}{ll}29 & 2\end{array}$ & 27 & 33 & 34 & 39 & 35 & 34 & 38 & 35 & 40 & 39 & 39 & 34 & 37 & 33 & 45 & 25 \\
\hline & & & & & & & & & & 16 & & & & & & & & & \\
\hline & & & & & & & & & & ragr & & & & & & & & & \\
\hline IG & URA & 4. ( & Cont & ti & & & & & & & & & & & & & & & \\
\hline
\end{tabular}

vera, se reducen las diferencias entre los distintos tramos del río (Fig. 3). Durante esta época las comunidades más densas se recogieron en la estación 7 (13.080 individuos $\left./ \mathrm{m}^{2}\right)$. La mayor variación temporal se observa en las estaciones 3, 5 y 9 (t-test, p<0.01).

La Fig. 4 muestra la distribución espacial en ambas épocas de los 84 taxones identificados. En ella puede apreciarse de una forma más detallada las tendencias espaciales y temporales de las 32 unidades taxonómicas más frecuentes y abundantes del conjunto total, así como las de algunos taxones abundantes cuya distribución se encuentra restringida a un tramo concreto del río. De los demás taxones unicamente se apunta su presencia/ausencia. Algunos, como oligoquetos, simúlidos, quironómidos y coleópteros élmidos de los géneros Elmis, Esolus y Limnius, aunque abundantes en ambas épocas, fueron sensiblemente más numerosos durante el otoño. Otros experimentaron importantes cambios estacionales en densidad, como Ancylus fluviatilis, Atherix, Hydropsyche, Sericostomatidae, Polycentropus y Psychomyiidae, especialmente abundantes durante el otoño y escasa en primavera. Por el contrario, Ephemerella ignita, Helodidae y Agapetinae incrementaron su densidad durante la primavera. Puede observarse también una segregación espacial de algunos taxones como Dugesia, Echinogamrnarus, heptagénidos de los géneros Epeorus, Rhithrogena y Ecdyonurus y coleópteros élmidos de los géneros Elmis, Esolus y Limnius, que alcanzan elevadas densidades en el tramo medio-bajo del río (estaciones 6, 7 y 8). En este mismo tramo se aprecia una disminución en la densidad de oligoquetos, quironómidos e hidropsíquidos.

En otoño se observó un acusado descenso del número de taxones entre las estaciones 2 y 3. En primavera, la estación 7, con 45 taxones presentó la máxima riqueza, apreciándose un empobrecimiento de la comunidad en la estación 9, donde unicamente se identificaron 25 (Fig. 4).

Cada uno de los taxones identificados tiene asignada en la Fig. 4 una categoría trófica según su modo de alimentación (CUMMINS, 1974; MERRITT \& CUMMINS, 1978; CUMMINS \& KLUG, 1979). La Fig. 5 muestra la importancia relativa de cada uno de los grupos funcionales a lo largo del río, en ambas épocas. En general, los recolectores y los generalistas (quironómidos) alternan la dominancia de las comunida-
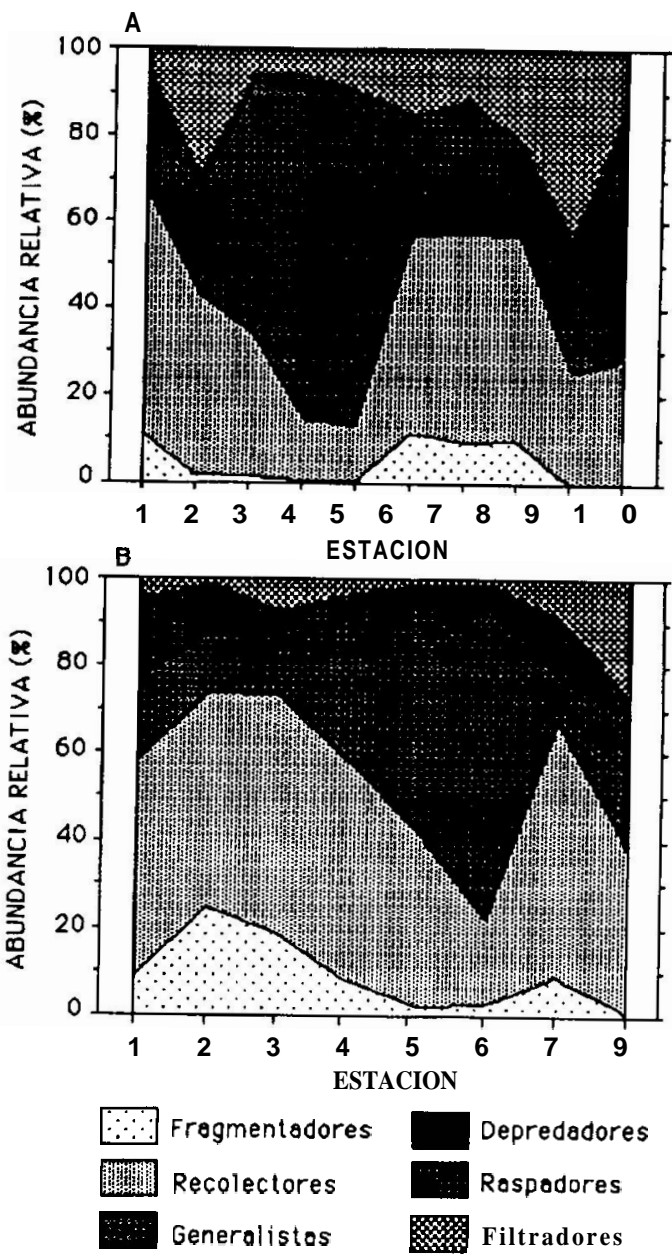

FIGURA 5. Abundancia relativa de los grupos funcionales a lo largo del no. A, otoño. B, primavera.

FIGURE 5. Relative abundance of functional groups along the stream. A, autumn. B, spring.

des de macroinvertebrados en distintos tramos del río Agüera. El grupo de los recolectores está dominado principalmente por taxones como oligoquetos, efemerópteros heptagénidos (Epeorus, Rhithrogena y Ecdyonurus), Caenis, Baetis, 
Leptoceridae y coleópteros élmidos (Elmis, Esolus y Limnius). Durante el otoño (Fig. 5A), las diferencias espaciales de los recolectores se hacen máximas entre las estaciones 4 y 5 y la 1 (ANOVA, $\mathrm{p}<0.01$ ), mientras que en el caso de los generalistas los extremos corresponden a las estaciones 7 y 8 y la 4 (ANOVA, $\mathrm{p}<0.01$ ). Este esquema cambia durante la primavera (Fig. $5 \mathrm{~B}$ ), dándose las mayores diferencias de recolectores entre las estaciones 6 y 7 (ANOVA, p<0.01). En el caso de los generalistas, su menor importancia sigue registrándose en la estación 7 (ANOVA, $\mathrm{p}<0.01$ ).

Por su parte, las máximas diferencias espaciales para los filtradores, constituídos principalmente por simúlidos e hidropsíquidos, se establecen entre la estación 1, donde se registra el mínimo, y la 9, donde se observa el máximo (ANOVA, p $<0.05$ ) en otoño, y entre la 2 (mínimo) y la 9 (máximo) (ANOVA, p<0.01) en primavera.

La importancia relativa de los fragmentadores, dominados por Echinogammarus, Leuctra, Nemoura y Sericostomatidae, es mínima en la estación 9 y máxima en el tramo 6-8 durante el otoño (ANOVA, $\mathrm{p}<0.01$ ), mientras que en primavera los extremos corresponden a la estación 9 y al tramo 2-3 (ANOVA, $\mathrm{p}<0.01$ ), en el mismo sentido.

Los raspadores del substrato, en otoño, muestran su mayor importancia relativa en el tramo medio-bajo del río (estaciones 6, 7 y 8), mientras que su mínima corresponde a los tramos superiores (ANOVA, p<0.01). En primavera, la máxima se da en la estación 7, y la mínima en la 3 (ANOVA, p<0.01). Dentro de este grupo, Potamopyrgus jenkinsi y Ancylus fluviatilis eran especialmente importantes en otoño, mientras que Ephemerella ignita y Helodidae incrementaban su importancia durante la primavera.

TABLA 2. Significado ( $\mathrm{t}$ test, $\mathrm{p}<0,05)$ de los cambios temporales en la abundancia relativa de cada grupo trófico en cada estación. O (otoño) y P (primavera) indican mayores valores en esa época. N.S.=diferencias no significativas. $\mathrm{CR}=$ recolectores, $\mathrm{G}=$ generalistas, $\mathrm{F}=$ fragmentadores, $\mathrm{R}=$ raspadores, $\mathrm{CF}=$ colectores-filtradores, $\mathrm{D}=$ depredadores.

TABLE 2. Significance of seasonal changes ( $t$ test, $p<0,05)$ in the relative abundance of each functional group at each site. O (autumn) and $\mathrm{P}$ (spring) mean higher values in this season. N.S.= no significant differences. $\mathrm{CR}=$ collector-gatherers, $\mathrm{G}=$ generalists, $\mathrm{F}=$ shredders, $\mathrm{R}=$ scrapers, $\mathrm{CF}=$ collector-filterers, $\mathrm{D}=$ predators.

\begin{tabular}{ccccccc} 
Estación & CR & $\boldsymbol{G}$ & $\mathbf{F}$ & R & CF & D \\
\hline 1 & NS & NS & NS & NS & NS & NS \\
2 & NS & NS & P & P & O & P \\
3 & P & O & P & P & NS & P \\
4 & P & O & P & P & NS & NS \\
5 & P & O & P & P & O & O \\
6 & O & P & O & NS & O & NS \\
7 & NS & NS & NS & NS & NS & NS \\
9 & P & NS & P & NS & NS & NS \\
\hline
\end{tabular}

Los depredadores estaban dominados principalmente por ácaros, Atherix y Rhyacophila. En general, su importancia relativa era escasa, pero significativamente mayor en las estaciones 1 y 5 que en las 9 y 10 en otoño (ANOVA, p<0.01). En primavera, su mayor importancia ocum'a en la 1 frente a la mínima de la 9 (ANOVA, p<0.01).

Por lo que respecta a los cambios temporales que operan en la abundancia relativa de cada grupo trófico para cada estación, en la Tabla 2 se ha resumido la significación de tales cambios. En líneas generales, las estaciones 1, 7 y 9 son las que presentan una estructura de comunidad sujeta a menor cambio. Por su parte, la estación más cambiante es la 5 , en consonancia con la mayor variabilidad general observada en los tramos medios. Los recolectores, fragmentadores y raspadores del substrato, en las estaciones donde cambian de una época a otra y salvo alguna excepción, suelen aumentar en primavera (Tabla 2). Por su parte, generalistas y filtradores, del mismo modo, disminuyen sus proporciones en esta época.

\section{DISCUSIÓN}

El corto recorrido de los ríos del norte de la Península Ibérica conlleva que, en tan solo unos pocos kilómetros, la cuenca fluvial concentre un mosaico de condiciones (substrato, vegetación riparia, actividades humanas) que pueden inducir cambios en la distribución y abundancia de las comunidades de macroinvertebrados bentónicos. El río Agüera no es una excepción a esta situación. Sin embargo, el grado de perturbación humana que presenta es inferior al de otros ríos del entorno geográfico (ORIVE et al., 1989), y "a prior" son esperables variaciones de menor magnitud.

Como en otros sistemas fluviales (DUDGEON, 1984; EDWARDS \& BROOKER, 1984; WARD, 1986), el efecto del incremento de la densidad de macroinvertebrados río abajo también es observado en el río Agüera, en situaciones de estabilidad hidrológica, como indican los resultados encontrados durante el otoño. Sin embargo, esa tendencia longitudinal se mitiga en situaciones de mayor caudal, dado el escaso tiempo de residencia del agua, y la homogenización que opera en el río tras una riada. Tal es el caso registrado durante la primavera en el río Agüera. Un segundo efecto lo constituye la reducción de abundancia de macroinvertebrados, no necesariamente asociado a una reducción de taxones. El ciclo hidrplógico condiciona la densidad de macroinvertebrados, como se ha puesto de manifiesto en numerosos trabajos (ej. BUNN et al., 1986; RESH et al., 1988; GRIMM \& FISHER, 1989; REICE et al., 1990). Si bien algunos taxones como Dugesia, Ancylus fluviatilis, Nemoura, Atherix, Hydropsyche, Sericostomatidae, 
Polycentropus y Psychomyiidae disminuyen mucho su abundancia en primavera, otros adquieren mayor desarrollo que durante el otoño, como Ephemerella ignita, Hidraenidae, Dupophilus, Helichus, Ceratopogonidae y Agapetinae.

Los caudales elevados pueden producir una mayor similitud espacial en la composición de la comunidad, como sucede en otros sistemas fluviales de la comisa cantábrica (ABELLA \& GONZÁLEZ, 1986). En el Agüera, la situación de mayor caudal genera que algunos taxones se distribuyan más ampliamente a lo largo del río pues las perturbaciones causadas por las crecidas tienden a redistribuir el bentos fluvial (CULP \& DAVIES, 1982; TOWNSEND, 1989). Sin embargo, los resultados obtenidos no indican una mayor homogeneidad entre estaciones en cuanto al número de taxones encontrado, ni tampoco se observan cambios relevantes en la riqueza taxonómica de una época a otra.

El análisis de las comunidades de macroinvertebrados en términos de grupos funcionales puede tener mayor significado que el de las entidades taxonómicas cuando se estudia el río desde un punto de vista funcional (CUMMINS, 1988). El río Agüera está dominado por los recolectores y por los generalistas, fundamentalmente quironómidos, considerados en esta categoría dada la variedad de formas de alimentación que presentan diferentes componentes de esta familia (MERRITT \& CUMMINS, 1978; HACHMÖLLER et al., 1991). Los recolectores, que aprovechan principalmente el material particulado fino del sustrato (CUMMINS, 1973), denotan la importancia de este tipo de recurso derivado de la fragmentación y procesado de los aportes alóctonos en una cuenca con importantes masas forestales como la del Agüera. Ambos grupos alternan su importancia relativa a lo largo del perfil longitudinal, siendo favorecidos los generalistas en los tramos más humanizados (pequeños núcleos urbanos de la cuenca). Estas diferenciaciones espaciales aparecen más marcadas durante la época de mayor estabilidad hidrológica.

Los fragmentadores han mostrado una nítida segregación espacial relacionada con los tramos forestados (cabecera y tramos medios), donde los aportes directos de materia orgánica particulada gruesa son mayores. Esta relación ha sido sugerida o puesta de manifiesto en numerosos trabajos (HAWKINS \& SEDELL, 1981; CULP \& DAVIES, 1982; DUDGEON, 1984; IVERSEN, 1988; CUMMINS et al., 1989). Según el RCC (VANNOTE $\boldsymbol{e t} \boldsymbol{a l} .$, 1980) los fragmentadores se encontrarían relegados a tramos altos forestados, aunque existen resultados discrepantes (MARCHAN et al., 1985, WINTERBOURN $\boldsymbol{e t}$ al., 1981). Nuestros datos sugieren una estrecha relación de este grupo con la vegetación riparia, pero asociada también con una escasa eutrofia de las aguas, pues al nivel de la estación 9, en una situación de aguas más eutrofizadas (ELOSEGUI \& POZO, 1992), aún manteniéndose una buena cobertura arbórea riparia, los fragmentadores son muy escasos. En este sentido, DUDGEON (1984) observó que la eutrofización cultural elimina las poblaciones de fragmentadores. Algunos de los taxones incluídos en este grupo son poco tolerantes a los descensos de la calidad de las aguas como Leuctra, Nemoura y Sencostomatidae (PALAU \& PALOMES, 1986; BARGOS et al., 1990).

Los raspadores del substrato alcanzan su mayor importancia relativa en el tramo 6-8, coincidiendo con el mayor desarrollo de los productores primarios (datos no publicados) en un tramo de autodepuración del río (ELOSEGUI \& POZO, 1992), y en consonancia con la utilización directa de las algas como alimento (CUMMINS, 1973). En teoría y según las condiciones de luz y temperatura, la primavera es una época más propicia para el desarrollo del perifiton. Sin embargo, la mayor densidad de estos macroinvertebrados se ha obtenido en otoño, lo cual podría ser explicado como consecuencia de la estabilidad hidrológica (bajo caudal) y moderadas temperaturas ocumdas en esa época, que alargaron las condiciones estivales, favoreciendo el desarrollo de las algas. Los raspadores han presentado un significativo cambio de composición de una época a otra. Mientras en otoño lo constituyen fundamentalmente Potamopyrgus jenkinsi y Ancylus fluviatilis, en primavera estaba dominado por Ephemerella ignita y Helodidae, lo que indica una segregación temporal dentro del grupo que utiliza directamente los productores primarios como recurso. El desarrollo importante de Potamopyrgus jenkinsi durante el otoño, ha sido comentado ya por BARGOS et al. (1990) en otros ríos del entorno. Asimismo, PUIG (1983) y DOLEDEC (1989) han señalado la estacionalidad que presenta Ephemerella ignita durante la primavera en ríos mediterráneos.

El mayor significado de los filtradores (constituídos principalmente por simúlidos e hidropsíquidos) se produce a nivel del tramo más eutrofizado (estación 9) y donde la concentración de materia orgánica particulada fina se mantiene más frecuentemente elevada (POZO et al., 1993). Diferentes autores han observado un incremento de las larvas filtradoras de Hydropsyche en tramos bajos de los ríos, que han explicado en función de un incremento de la concentración de materia orgánica en suspensión (GARCÍA DE JALÓN, 1986; PETERSEN, 1987; BASAGUREN \& ORIVE, 1991; CORTES, 1992). La situación concreta en los tramos finales del Agüera debe responder más a las condiciones de perturbación de las aguas por las actividades humanas que a una segregación natural en el río de los grupos funcionales, ya que el sistema que nos ocupa estaría en su totalidad enmarcado en lo que son los tramos altos de otros sistemas fluviales de mayor recorrido y donde los gru- 
pos dominantes deberían corresponder a los fragmentadores y recolectores (VANNOTE et al., 1980).

Coincidiendo con DUDGEON (1984), hemos observado que los depredadores constituyen una pequeña parte de la comunidad béntica y mantienen su abundancia relativa sin grandes cambios en todas las secciones del río.

Finalmente, en comparación con otros ríos del entorno geográfico, con niveles superiores de perturbación humana y mayor diferenciación espacial en la composición de las comunidades fluviales (BARGOS et al., 1990; BASAGUREN \& ORIVE, 1990) el río Agüera muestra una variabilidad en las comunidades de macroinvertebrados a lo largo del gradiente fluvial que aparece más marcada en situaciones de escaso caudal, cuando se potencian los procesos productivos del río y se acentúa la eutrofización en ciertas zonas como consecuencia de la menor dilución.

\section{AGRADECIMIENTOS}

Este trabajo está extraído de un proyecto más amplio financiado por la Universidad del País Vasco/E.H.U. (U.P.V. 118.310 - 0067188).

Agradecemos a T. Bargos, A. Rubio y A. Uriarte, su colaboración en el análisis de las muestras.

\section{BIBLIOGRAFÍA}

ABELLA, M.A. \& M.J. GONZALEZ, 1986. Variación estacional de la fauna dulceacuícola del alto Nalon, Asturias. Limnética, 2:173-179.

BARGOS, D., J.M. MESANZA, A. BASAGUREN \& E. Orive, 1990. Assessing river water quality by means of multifactorial methods using macroinvertebrates. A comparative study of main water courses of Biscay. Wat. Res., 24:1-10

BASAGUREN, A. \& E. ORIVE, 1990. The relationship between water quality and caddisfly assemblage structure in fast running rivers. The river Cadagua basin. Environ. Monit. Assess., 15:35-48.

BASAGUREN, A. \& E. ORIVE, 1991. Los insectos tricópteros como indicadores de la calidad del agua de los ríos de Bizkaia. Cuenca del Nerbion. Kobie, 20:39-44.

BUNN, S.E., D.H. EDWARD \& N.R. LONERAGAN, 1986. Spatial and temporal variation in the macroinvertebrate fauna of streams of the northern jarrah forest, Western Australia: community structure. Freshwat. Biol., 16:67-91.

CORTES, R.M.V., 1992. Seasonal pattern of benthic communities along the longitudinal axis of river systems and the influence of abiotic factors on the spatial structure of those communities. Arch. Hydrobiol., 126:85-103.

CULP, J.M. \& R.W. DAVIES, 1982. Analysis of longitudinal zonation and the river continuum concept in the OldmanSouth Saskatchewan river system. Can. J. Fish. Aquat. Sci., 39:1258-1266.

CUMMINS, K.W., 1973. Trophic relations of aquatic insects. Ann. Rev. Entomol., 18:183-206.

CUMMINS, K.W., 1974. Structure and function of stream ecosystems. BioScience, 24:631-641.

CUMMINS, K.W. \& M.J. KLUG, 1979. Feeding ecology of stream invertebrates. Ann. Rev. Ecol. Syst., 10:147-172.

CUMMINS, K.W., 1988. The study of stream ecosystems: a functional view. In: Ecosystem Processes. (L.R. POMEROY \& J.J. ALBERTS, eds.) 240-254. Springer-Verlag. New York.

CUMMINS, K.W., M.A. WILZBACH, D.M. GATES, J.B. PERRY \& W.B. TALIAFERRO, 1989. Shredders and riparian vegetation. BioScience, 39:24-30.

DOLEDEC, S., 1989. Seasonal dynamics of benthic macroinvertebrate communities in the Lower Ardèche River (France). Hydrobiología, 182:73-89.

DUDGEON, D. 1984. Longitudinal and temporal changes in functional organization of macroinvertebrate communities in the Lam Tsuen River, Hong Kong. Hydrobiología, 111:207217.

EDWARDS, R.W. \& M. BROOKER, 1984. Wye. In: Ecology of European rivers, (B.A. WHITTON, ed.) 51-82. Blackwell Scientific Publications. Oxford.

ELOSEGUI, A. \& J. POZO, 1992. Physico-chemical characteristics of the Agüera River (Spain), during an unususal hydrologically period. Annls. Limnol., 28:85-96.

EUSKOIKER, 1988. Estudio hidrobiológico de la red hidrográfica de Bizkaia. Diputación Foral de Bizkaia. Informe final, Bilbao.

GARCÍA DE JALÓN, D., 1986. Los Hydropsychidae (Trichoptera) de la cuenca del Duero. Boletín Asoc. esp. Entom., 10:127-138.

GRIMM, N.B. \& S.G. FISHER, 1989. Stability of periphyton and macroinvertebrates to disturbance by flash floods in a desert stream. J. N. Am. Benthol. Soc., 8:293-307.

HACHMÖLLER, B., R.A. MATTHEWS \& D.F. BRAKKE, 1991. Effects of riparian community structure, sediment size, and water quality on the macroinvertebrate communities in a small, suburban stream. Northwest Sci., 65:125-132.

HART, D.D., 1983. The importance of competitive interactions within stream population and communities. In: Stream Ecology (J.R. BARNES \& G.W. MINSHALL, eds.) 99-136. Plenum Press. New York. 
HAWKINS, C.P. \& J.R. SEDELL, 1981. Longitudinal and seasonal changes in functional organization of macroinvertebrate communities in four Oregon streams. Ecology, 62:387-397.

IVERSEN, T.M. 1988. Secondary production and trophic relationships in a spring invertebrate community. Limnol. Oceanogr., 3:582-592.

MARCHANT, R., L. METZELING, A. GRAESSER \& P. SUTER, 1985. The organization of macroinvertebrate communities in the major tributaries of the La Trobe river, Victoria, Australia. Freshwat. Biol., 15:315-331.

MERRITT, R.W. \& K.W. CUMMINS, 1978. An introduction to the aquatic insects of North America. Kendall \& Hunt Dubique, Iowa.

ORIVE, E., A. BASAGUREN, B. BIKUÑA \& M. CACHO, 1989. A comparative study of water mineralization and eutrophication in main water courses of Biscay (Basque Country) by means of factorial analysis. Wat. Res., 23:705-710.

OUTRIDGE, P.M., 1988. Seasonal and spatial variations in benthic macroinvertebrate communities of Magela Creek, Northern Territory. Aust. J. Mar. Freshwat. Res., 39:211-223.

PALAU, A. \& A. PALOMES, 1986. Los macroinvertebrados bentónicos como elementos de juicio para la evaluación de la calidad biológica del río Segre (Lérida, NE España). Limnética, 2:205-215.

PECKARSKY, B.L., S.C. HORN \& B. STATZNER, 1990. Stonefly predation along a hydraulic gradient: a field test of the harsh-benign hypothesis. Freshwat. Biol., 24:181-191.

PETERSEN, R.C.Jr., 1987. Seston quality as a factor influencing Trichopteran population. Proc. 5th Int. symp. on Trichoptera (M. BOURNAUD \& H. TACHET, eds.) 287-292. Junk. The Hague.

POZO, J., A. ELOSEGUI \& A. BASAGUREN, 1993. Seston transport variability at different spatial and temporal scales in the Agüera watershed (North Spain). Wat. Res., (en prensa).

PUIG, M.A., 1983. Efemerópteros y plecópteros de los ríos catalanes. Tesis Doctoral, Barcelona.

REICE, S.R., R.C. WISSMAR \& R.J. NAIMAN, 1990. Disturbance regimes, resilience, and recovery of animal communities and habitats in lotic ecosystems. Environ. Manag., $14: 647-659$

RESH, V.H., A.V. BROWN, A.P. COVICH, M.E. GURTZ, H.W. LI, G.W. MINSHALL, S.R. REICE, A.L. SHELDON, J.B. WALLACE \& R.C. WISSMAR, 1988. The role of disturbance in stream ecology. J. N. Am. Benthol. Soc., 7:433-495.

RICHARDSON, J.S., 1991. Seasonal food limitation of detritus in a montane stream: an experimental test. Ecology, $72: 873-887$.
ROSILLON, D., 1989. The influence of abiotic factors and density-dependent mechanisms on between-year variations in a stream invertebrate community. Hydrobiología, 179:25-38.

TOWNSEND, C.R., 1989. The patch dynamics concept of stream community ecology. J. N. Am. Benthol. Soc., 8:36-50.

VANNOTE, R.L., G.W. MINSHALL, K.W. CUMMINS, J.R. SEDELL \& C.E. CUSHING, 1980. The river cohtinuum concept. Can. J. Fish. Aquat. Sci., 37:130-137.

WARD, J.V., 1986. Altitudinal zonation in a Rocky Mountain stream. Arch. Hydrobiol., Suppl. 74:133-199.

WARD, J.V., 1992. Aquatic insect ecology. $l$. Biology and habitat. John Wiley \& Sons, Inc. New York.

WINTERBOURN, M.J., J.S. ROUNICK \& B. COWIE, 1981. Are New Zealand stream ecosystems really different? NZJ. Mar. Freshwat. Res., 15:321-328.

ZAR, J.H. 1984. Biostatistical Analysis. Prentice-Hall Intemational, Inc. New Jersey.

\begin{tabular}{llll}
\hline APÉNDICE & & \\
Significado del código de los taxones de la Fig. 4. \\
ACAR & Hidracarina & HCHE & Hydropsychidae \\
AGAP & Agapetinae & HELD & Helodidae \\
AMPH & Amphinemura & HELI & Helichus substriatus \\
ANCY & Ancylus fluviatilis & HELO & Helobdella \\
ANTH & Anthomyidae & HELP & Helophorus \\
APHE & Aphelocheirus montandoni & HIDR & Hidraenidae \\
ASEL & Asellidae & HSIL & Hydropsychesiltalai \\
ATHE & Atherix & ISOP & Isoperla \\
ATRI & Atrichops & LEPC & Leptoceridae \\
BAET & Baetis & LEPI & Lepidostomatidae \\
BLEP & Blephariceridae & LEPT & Leptophlebidae \\
BOYE & Boyeria irene & LEUC & Leuctra \\
BRAC & Brachycentridae & LIMN & Limnius \\
BYTH & Bythinella & LIMO & Limoniidae \\
CAEN & Caenis & LIMP & Limnephilidae \\
CERA & Ceratopogonidae & LYMN & Lymnaea \\
CHEU & Cheumatopsychelepida & NEMO & Nemoura \\
CHIM & Chimarra marginata & ODON & Odontocerum albicome \\
CHIR & Chironomidae & OLIG & Oligochaeta \\
CORI & Conxidae & ONYC & Onychogomphus \\
DIXI & Dixidae & OREC & Orectochilus \\
DOLI & Dolichopodidae & OULI & Oulimnius \\
DRYO & Dryops & PCHO & Psychomyiidae \\
DUGE & Dugesia & PCTP & Polycentropus \\
DUPO & Dupophilusbrevis & PERL & Perlamarginata \\
DYTI & Dytiscidae & PHIL & Philopotamus \\
ECDY & Ecdyonurus & PISI & Pisidium \\
ECHI & Echinogammarus & PLEC & Plectrocnemia \\
& & & \\
& & & \\
& & &
\end{tabular}




\begin{tabular}{|c|c|c|c|c|c|c|c|}
\hline \multicolumn{8}{|c|}{ APÉNDICE (Continuación) } \\
\hline ELMI & Elmis & POLY & Polycelis & GLOF & Glossiphonia & SIPH & Siphonoperla \\
\hline EMPI & Empididae & POTA & Potamopyrgus jenkinsi & GLOS & Glossosomatidae & STEN & Stenelmis \\
\hline EPEO & Epeorus & PROT & Protonemura & GOER & Goeridae & STRA & Stratiomyidae \\
\hline EPHE & Ephemera & PSYC & Psychodidae & HABR & Habrophlebia & TABA & Tabanidae \\
\hline EPHL & Ephemerella ignita & RHIT & Rhithrogena & HAEM & Haemopis sanguisuga & TILA & Hydroptila \\
\hline ERPO & Erpobdella & RHYA & Rhyacophila & HALI & Haliplus & TIPU & Tipulidae \\
\hline ESOL & Esolus & SERI & Sencostomatidae & & & & \\
\hline EULE & Euleuctra & SIMU & Simuliidae & & & & \\
\hline
\end{tabular}

